DE

M E D I C I N A

T R O P I C A L

$\mathrm{DE}$

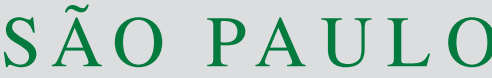

JOURNAL OF THE SÃO PAULO INSTITUTE OF TROPICAL MEDICINE

'Universidad Autonoma de Yucatan, Centro de Investigaciones Regionales "Dr. Hideyo Noguchi”, Laboratorio de Arbovirologia, Merida, Yucatan, Mexico

2Instituto Nacional de Salud Publica, Centro Regional de Investigación en Salud Publica Tapachula, Chiapas, Mexico

${ }^{3}$ Instituto Nacional de Medicina Tropical, Puerto Iguazú, Misiones, Argentina

${ }^{4}$ Universidad Juarez Autonoma de Tabasco, Laboratorio de Enfermedades Tropicales y Transmitidas por Vector, Tabasco, Mexico

${ }^{5}$ Universidad Internacional SEK, Facultad de Ciencias Naturales y Ambientales, Laboratorio de Biodiversidad y Salud Ambiental, Quito, Ecuador

Correspondence to: Carlos Marcial BaakBaak

Universidad Autonoma de Yucatan, Centro de Investigaciones Regionales "Dr. Hideyo Noguchi”, Laboratorio de Arbovirologia, Calle $96 \mathrm{~s} / \mathrm{n}$ x Av. Jacinto Canek y 47 Fraccionamiento Paseo de las Fuentes, 97225, Mérida, Yucatán, México

E-mail: carlos.baak@correo.uady.mx

Received: 26 April 2018

Accepted: 28 June 2018

\section{Study of Aedes aegypti population with emphasis on the gonotrophic cycle length and identification of arboviruses: implications for vector management in cemeteries}

Julian E. Garcia-Rejon', Armando Ulloa-Garcia², Nohemi Cigarroa-Toledo', Angelica Pech-May ${ }^{3}$, Carlos Machain-Williams' ${ }^{1}$, Rosa Carmina Cetina-Trejo', Lourdes Gabriela Talavera-Aguilar ${ }^{1}$, Oswaldo Margarito Torres-Chable ${ }^{4}$, Juan Carlos Navarro ${ }^{5}$, Carlos Marcial Baak-Baak ${ }^{1}$

\section{ABSTRACT}

Aedes aegypti is the vector of the arboviruses causing dengue, chikungunya and zika infections in Mexico. However, its presence in public places has not been fully evaluated. In a cemetery from Merida, Yucatan, Mexico, the productivity of Ae. aegypti, the gonotrophic cycle, and the presence of Ae. aegypti females infected with arboviruses were evaluated. Immature and adult mosquitoes were inspected every two months between April 2016 to June 2017. For the gonotrophic cycle length, the daily pattern of total and parous female ratio was registered and was analyzed using time series analysis. Ae. aegypti females were sorted into pools and assayed for flavivirus RNA by RT-PCR and Sanger sequencing. Aedes aegypti immatures represented $82.86 \%(8,627 / 10,411)$ of the collection. In total, 1,648 Ae. aegypti females were sorted into 166 pools. Two pools were positive; one for dengue virus (DENV-1) and the other for zika virus (ZIKV). The phylogenetic analysis revealed that the DENV-1 is more closely related to isolates from Brazil. While ZIKV is more closely related to the Asian lineage, which were isolates from Guatemala and Mexico. We report some evidence of vertical transmission of DENV-1 in nulliparous females of Ae. aegypti. The gonotrophic cycle was four and three days in the rainy and dry season, respectively. The cemetery of Merida is an important focus of Ae. aegypti proliferation, and these environments may play a role in arboviruses transmission; probably limiting the efficacy of attempts to suppress the presence of mosquitoes in domestic environments.

KEYWORDS: Arbovirus. Dengue. Mexico. Daily survival. Zika virus.

\section{INTRODUCTION}

Cemeteries are obligatory components of human settlements. In Latin American cultures, cemeteries are important places to honor the deads, and it is common to have a large influx of visitors to cemeteries throughout the year ${ }^{1}$. Previous studies revealed that cemeteries are suitable habitats for mosquitoes due to the great availability of resources such as sugar containing substances, shelter and water-filled vases ${ }^{2,3}$. Immature stages of Aedes aegypti are common in cemeteries, where larvae and pupae are often found inside vases ${ }^{1,3}$. However, few studies have quantified the adult populations and their role in the arboviruses transmission ${ }^{2}$. To the best of our knowledge, there are only two reports of arboviruses identified in mosquitoes collected in cemeteries ${ }^{4,5}$. Therefore, it is important to know the epidemiological importance of cemeteries in areas where dengue, zika and chikungunya viruses are present. 
In home environments, survivorship and gonotrophic cycle of Ae. aegypti are well-documented ${ }^{6}$. A short time of the gonotrophic cycle of Ae. aegypti increases the contact vector-human and thus the risk for arbovirus transmission ${ }^{7}$. Previous studies carried out in houses estimated between 3 to 7 days the gonotrophic cycle of Ae. aegypti; the region, season, and temperature affected significantly the cycle $e^{6,8,9}$. It has also been observed that mosquitoes can disperse beyond the houses. Previous studies reported engorged Ae. aegypti in schools and churches ${ }^{10,11}$. Therefore, the vectorial capacity of mosquitoes must be evaluated in cemeteries because they have breeding sites and are near the houses.

Cemeteries have been used to study the mosquito ecology (i.e., productivity, species interaction, competition, composition and temporality), and also in field assays to evaluate biological and chemical mosquito control ${ }^{1,12,13}$. Cemeteries have also been used for the early detection and monitoring of invasive mosquitoes such as Aedes albopictus (Skuse) $)^{14}$. There is a growing recognition that cemeteries can also be effective sites for monitoring virus transmitted by mosquitoes ${ }^{4,5}$. In Yucatan State of Southeastern Mexico, dengue, chikungunya and zika viruses co-occur ${ }^{15,16}$. Despite this, studies have not been performed to quantify the Ae. aegypti population in cemeteries of Merida city and whether they are potential sites for arboviruses transmission. The goals of the study were to determine by season 1) the infestation of breeding sites; 2) abundance of immatures and adults of Ae. aegypti; 3 ) the length of the gonotrophic cycle and the survival rate of Ae. aegypti; and 4) the presence of Ae. aegypti females infected with arbovirus.

\section{MATERIALS AND METHODS}

\section{Study area}

The study was carried out in the "General Cemetery" of
Merida city in the Yucatan State of Southeastern Mexico. This cemetery is the oldest and largest (15 hectares), is immersed within a densely populated city. Based on data of the town hall, the cemetery has 25,700 vaults registered as tombs, ossuaries, niches, crypts and mausoleums (http:// www.merida.gob.mx/). The area selected for the study is located approximately $300 \mathrm{~m}$ from the nearest houses (Figure 1). The cemetery is open to public between 07:00 to $18: 00 \mathrm{~h}$.

In Yucatan State, the rainy season extends from May to October and the dry season from November to April. During the rainy season, the mean rainfall is $1,000 \mathrm{~mm}$ and the mean temperature of $27.5^{\circ} \mathrm{C}$. During the dry season, the mean rainfall is $300 \mathrm{~mm}$ and the mean temperature is $25.1{ }^{\circ} \mathrm{C}^{10}$.

\section{Adult mosquitoes collection}

Adult mosquitoes were collected for three consecutive days in April, June, August, October and December 2016 and in February, April and June 2017. Aedes aegypti females were collected using BG-Sentinel traps (Biogents $\mathrm{GmbH}$, Regensburg, Germany) coupled to the attractant BG-Lure (Biogents GmbH, Regensburg, Germany). Inside the cemetery, we chose a transect of $170 \mathrm{~m}$, in which ten traps were placed. The transect was located near the flower shop due to the influx of visitors and presence of cemetery workers (Figure 1). BG-Sentinel traps were placed at every $17 \mathrm{~m}$ and were activated between 07:00 and 10:00 h. Female Ae. aegypti were sorted into pools of up to 15 and stored at $-80{ }^{\circ} \mathrm{C}$ until required.

\section{Sampling of immature mosquitoes}

Mosquitoes were collected into a quadrant of approximately $100 \mathrm{~m}$, where the BG-Sentinel traps were placed. Mosquitoes were removed from vases using nets,

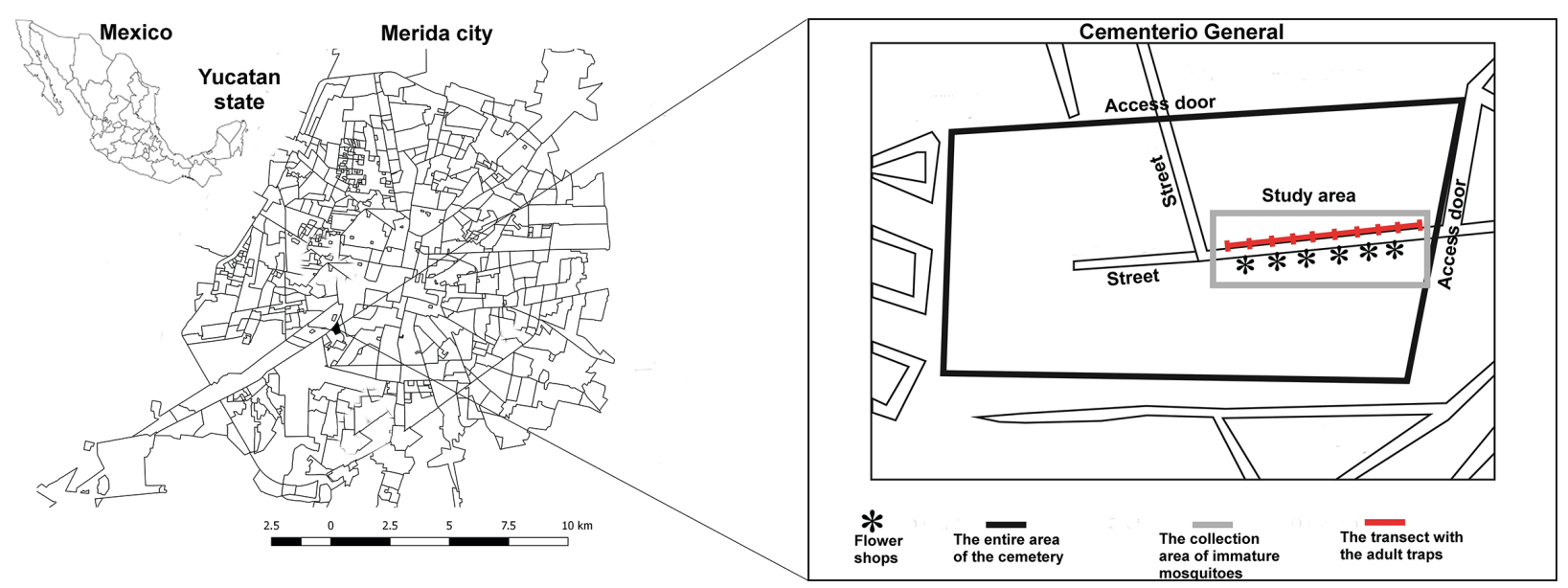

Figure 1 - Study area in the cemetery "Cementerio General" of Merida city, Yucatan, Mexico 
turkey basters and pipettes and placed inside plastic transportation containers labeled according to date, study site and sample identification number. Immature and adult mosquitoes were transported alive to the Laboratory of Arbovirology at Universidad Autonoma of Yucatan and were identified using published identification keys ${ }^{17,18}$.

\section{Gonotrophic cycle and survival dynamics}

Female Ae. aegypti were collected using BG-Sentinel traps during 19 consecutive days in the dry (April 20 to May 08) and rainy (September 06 to 24) season in 2016. The blood feeding status (Sella's stages) was determined by external examination of the abdomen. Insects were then grouped as unfed (the collapsed abdomen and the ovaries occupy one-third of the abdomen), fed (freshly fed, bright red blood and the ovaries occupy two to three segments ventrally; the sub-gravid with dark blood and with great space reduced and ovaries occupy most of abdomen) and gravid (blood completely digested or present only as a black trace or line $)^{10}$.

To estimate the gonotrophic cycle, all the females were dissected in microscope slides using a drop of $65 \%$ saline solution. They were classified as nulliparous, parous or gravid according to the appearance of the tracheolar system and/or the presence of eggs in the abdomen ${ }^{19}$. Aedes aegypti females dissected were stored at $-80^{\circ} \mathrm{C}$ and assayed for flavivirus RNA.

\section{RNA extraction and RT-PCR}

Pools of female adult Ae. aegypti were placed into eppendorf microtubes containing $300 \mu \mathrm{L}$ of Liebovitz's L15 medium (Invitrogen, Carlsbad, CA, USA) and mechanically homogenized using sterile pestles. Homogenates were centrifuged at $10,000 \times \mathrm{g}$ for $10 \mathrm{~min}$ and supernatants were collected. Total RNA was extracted from an aliquot $(100 \mu \mathrm{L})$ of each supernatant using the RNeasy kit (QIAGEN, Valencia, CA, USA) and tested for flavivirus RNA by reverse transcription-polymerase chain reaction (RT-PCR) using flavivirus-specific primers (cFD2 and FS778) which amplify a 250 nucleotide region of the NS5 gene $^{20}$. RT-PCRs were performed in $25 \mu \mathrm{L}$ reaction volumes containing $2.5 \mu \mathrm{L}$ of total RNA, $2 \mu \mathrm{L} \mathrm{MgCL} 2$ at a concentration of $25 \mathrm{mM}, 2.5 \mu \mathrm{L}$ of $5 \mathrm{x}$ reaction buffer, 0.2 $\mu \mathrm{L}$ of dNTPs, $0.15 \mu \mathrm{L}$ Taq polymerase (Invitrogen ${ }^{\circledR}$ ), $0.5 \mu \mathrm{L}$ of each primer at a concentration of $10 \mathrm{mM}$. and $16.65 \mu \mathrm{l}$ $\mathrm{ddH} 2 \mathrm{O}$ was added to reach the final volume. Amplification conditions are as follows: an initial denaturation of $95^{\circ} \mathrm{C}$ for 1 minute, followed by 35 cycles each consisting of $1 \mathrm{~min}$ at $95{ }^{\circ} \mathrm{C}, 1.5 \mathrm{~min}$ at $75^{\circ} \mathrm{C}$, and $1 \mathrm{~min}$ at $72{ }^{\circ} \mathrm{C}$ and one cycle of extension for $7 \mathrm{~min}$ at $72{ }^{\circ} \mathrm{C}$. Amplicons were visualized on $2 \%$ agarose gels with $0.5 \mu \mathrm{g} / \mathrm{mL}$ of ethidium bromide using a Doc ${ }^{\mathrm{TM}} \mathrm{XR}+\mathrm{Gel}$ Documentation System. RT-PCR products were purified using the Zymoclean DNA recovery kit Cat (Zymo Research, Irvine, CA, USA) and sequenced using a 3500xL DNA sequencer (Applied Biosystems, Foster City, CA, USA).

\section{Data analysis}

Entomological indices were estimated: 1) the percentage of water-filled containers with immature Ae. aegypti presence (larvae, pupae, or both); and 2) a pupal index representing the percentage of containers with Ae. aegypti pupae present out of all containers with Ae. aegypti immatures presence.

To compare the number of immature and adult of Ae. aegypti by season, data were submitted to a normality test. A Mann-Whitney U test was used to compare the number of immature and Ae. aegypti females by season, because data did not meet the assumptions of normality and homogeneity of variances. The minimum infection rate (MIR) was calculated: (number of positive pools/ total specimens tested) $\mathrm{x} 1,000$. Statistical analysis was performed using the IBM SPSS Statistics version 22 software for Windows (IBM Corporation, Armonk, NY, USA), and results were considered significant when $P \leq 0.05$.

The length of the gonotrophic cycle was estimated using a cross-correlation analysis ${ }^{7}$ with the formula $M_{t}=P_{u} T_{(t-u)^{\prime}}$ where $M=$ the number of parous individuals captured on day $t ; T_{(t-u)}=$ the total number of females (nulliparous and parous) captured on day $t-u ; u=$ the length of the gonotrophic cycle; and $P=$ the survival rate per gonotrophic cycle, calculated from the slope in a regression model. The correlation coefficient $(r)$ for day 0 represented the correlation between $P$ and $T_{t}$ data pairs from mosquitoes captured on the same day (15 data pairs). The $r$ for day was obtained by pairing daily $P$ data with the corresponding $T$ data of 1 day before. Likewise, $r$ for each day 1 was obtained by pairing daily $P_{t}$ data with the corresponding $T_{t}$ data of 1 day before. The $r$ for day 2 was calculated by pairing daily $P t$ data with corresponding $T t$ data of 2 days before, and so on. It was assumed that a significant $r$ between the same series expressed a time delay $(u)$ equivalent to the gonotrophic cycle. The highest correlation coefficient and significance obtained after day $0(u=0)$ indicated the number of days per gonotrophic cycle, with descending peaks occurring at multiples of this interval.

To eliminate spurious cross correlations, data were filtered using an autoregressive process with a lag of 
1 day, with the formula $Z_{t}=X-\beta\left(X_{t-1}\right)$, where $Z t=$ is the filtered time series, $X_{t}=$ the time series to be filtered, and $\beta=$ the estimated auto-regressive parameter ${ }^{21}$. A significant correlation between 2 filtered time series $\left(M_{t}\right.$ and $X_{(t-u)}$ was assumed), and $r$ corresponded to a lag $u$ equivalent to the gonotrophic cycle, with regular peaks at the start of each cycle.

Daily survival rates $(p)$ were calculated from the parity rates using the formula $p=(P R)^{1 / C G}$, where $P R=$ the parity rate and $C G=$ the duration of the gonotrophic cycle ${ }^{22}$.

\section{Sequence analysis}

Sequences were manually aligned and edited using the Bioedit v.7.0.9 $9^{23}$ and the Mega v. $7^{24}$ softwares. The nucleotide sequences were translated into the corresponding amino acid counterparts using the translation tool of the ExPASy bioinformatic resource portal (http://web.expasy. org/ translate/) and compared to other sequences from the GenBank database using the Basic Local Alignment Search Tool (https://blast.ncbi.nlm.nih.gov/Blast.cgi). The alignment of the NS5 fragment and amino acid sequences was performed with Mega v. $7^{24}$. The DnaSP v.5.10 software ${ }^{25}$ was also used to analyze genetic variants. The similarity and identity were calculated using the MatGat software ${ }^{26}$. Genetic distances among variants were calculated using the Mega v.7 software ${ }^{24}$ with 10,000 random permutations.

\section{RESULTS}

\section{Immature mosquitoes collection}

The total number of container observations for the entire study was 4,867 (Table 1). Water was detected during $16.29 \%$ $(793 / 4,867)$ of the container observations and $22.95 \%$ (182/793) yielded immatures. In a quadrant of $100 \mathrm{~m}$, the density of positive vases was 112 and 70 during the dry and rainy season, respectively. A total of 10,411 immatures of four species were collected. The most abundant species was Ae. aegypti $(\mathrm{n}=8,627)$, followed by Culex quinquefasciatus Say $(\mathrm{n}=1,663)$, Culex nigripalpus Theobald $(\mathrm{n}=69)$, and
Culex coronator Dyar and Knab $(\mathrm{n}=52)$.

Immature Ae. aegypti represented $82.86 \%$ of the collection. Analysis of data at the species level revealed that there was no significant statistical difference between the number of immature Ae. aegypti per season $(Z=-0.142$, $P \geq 0.05$ ); although two-fold more immatures were collected in the rainy season. A total of 3,014 vases observations were made during the rainy season. Water was detected in $14.56 \%(439 / 3,014)$ of the container observations and $25.51 \%$ (112/439) yielded immatures (Table 1). The pupal index was calculated as $47.32 \%$ (53/112). During the dry season, 1,853 vases observations were made. Of these, $19.10 \%(354 / 1,853)$ revealed water and $19.77 \%(70 / 354)$ yielded immatures. The pupal index was calculated as $32.85 \%(23 / 70)$.

\section{Adult mosquitoes collection}

In total, 3,957 adult mosquitoes (2,198 males and 1,759 females) of four species were collected (Table 2). Of the females collected, the most abundant species was Ae. aegypti $(\mathrm{n}=1,648)$, followed by Aedes taeniorhynchus (Wiedemann) $(\mathrm{n}=77)$, Aedes trivittatus (Coquillet) ( $\mathrm{n}=17)$, and $C x$. quinquefasciatus $(\mathrm{n}=17)$.

A significant statistical difference was observed in the median number of Ae. aegypti females per season $(Z=-8.099, P \leq 0.05)$. Approximately eight-fold more females were collected during the rainy season $(\mathrm{n}=1,471)$ compared to the dry season $(\mathrm{n}=177)$ (Table 2). Of the 1,471 Ae. aegypti females collected during the rainy season, 1,210 were identified as unfed, 121 as fed and 140 as gravid (Table 2). In the dry season, 177 Ae. aegypti females were collected with 117 identified as unfed, 14 as fed and 46 as gravid.

\section{Length of Ae. aegypti gonotrophic cycle}

There was no significant correlation $(P \geq 0.05)$ observed between raw and filtered data in daily changes of parity rates over 19 days in females collected during the dry and rainy season. Following the criteria of Bockarie et al. ${ }^{27}$, the highest $r$-values are considered for the duration of the

Table 1 - Abundance of Ae. aegypti immatures by season in a cemetery from Merida city, Yucatan from April 2016 to June 2017

\begin{tabular}{lccccccc}
\hline \multirow{2}{*}{ Season } & \multicolumn{2}{c}{ Containers } & \multicolumn{2}{c}{ Total number Ae. aegypti collected } & \multicolumn{2}{c}{ Entomological index } \\
\cline { 2 - 8 } & $\begin{array}{c}\text { Total number } \\
\text { examined }\end{array}$ & $\begin{array}{c}\text { Number (\%) } \\
\text { with water }\end{array}$ & Larvae & Pupae & Total & $\begin{array}{c}\text { \% water-filled containers with } \\
\text { Ae. aegypti immatures present }\end{array}$ & $\begin{array}{c}\text { Pupal } \\
\text { index (\%) }\end{array}$ \\
\hline Dry & 1,853 & $354(19.10)$ & 3,084 & 193 & 3,277 & 19.77 & 32.85 \\
Rainy & 3,014 & $439(14.56)$ & 4,968 & 382 & 5,350 & 25.51 & 47.32 \\
\hline Total & 4,867 & 793 & 8,052 & 575 & 8,627 & 22.95 & 41.72 \\
\hline
\end{tabular}


Table 2 - Species composition and abundance of adult mosquitoes collected in a cemetery of Merida Yucatan, from April 2016 to June 2017

\begin{tabular}{|c|c|c|c|c|c|}
\hline \multirow[t]{2}{*}{ Species } & \multicolumn{2}{|c|}{ Total number adults collected } & \multicolumn{3}{|c|}{ Blood feeding status } \\
\hline & Males & Females & Unfed & Fed & Gravid \\
\hline \multicolumn{6}{|l|}{ Rainy season } \\
\hline Ae. aegypti & 1,969 & 1,471 & 1,210 & 121 & 140 \\
\hline Ae. taeniorhynchus & & 77 & 57 & 4 & 16 \\
\hline Ae. trivittatus & 1 & 17 & 10 & 4 & 3 \\
\hline Cx. quinquefasciatus & 1 & 3 & 1 & 1 & 1 \\
\hline Subtotal & 1,971 & 1,568 & 1278 & 130 & 160 \\
\hline \multicolumn{6}{|l|}{ Dry season } \\
\hline Ae. aegypti & 220 & 177 & 117 & 14 & 46 \\
\hline Cx. quinquefasciatus & 7 & 14 & 3 & 5 & 6 \\
\hline Subtotal & 227 & 191 & 120 & 19 & 52 \\
\hline Total & 2,198 & 1,759 & 1,398 & 149 & 212 \\
\hline
\end{tabular}

gonotrophic cycle. During dry season, a high correlation on days 2, 5 and 8 was found, suggesting a gonotrophic cycle of 3 days (Table 3 ). A daily survival rate of 0.83 and parity rate of 0.58 (Table 4) in a mean temperature of $29.43 \pm 2.41^{\circ} \mathrm{C}$, $57.16 \pm 5.56 \% \mathrm{HR}$ and $2.03 \mathrm{~mm}$ of precipitation were estimated. During the rainy season, a high correlation on days 5, 9 and 13 was found suggesting a gonotrophic cycle of 4 days (Table 3). A daily survival rate of 0.89 and parity rate of 0.61 (Table 4 ) in a mean temperature of $26.7 \pm 1.22{ }^{\circ} \mathrm{C}, 79.44 \pm 5.64 \% \mathrm{HR}$ and $59.68 \mathrm{~mm}$ of precipitation were estimated. The daily survival rate was similar in dry (0.83) and rainy (0.89) seasons, and as a consequence, there was no significant difference between parity rate by season $(t=-1.596, d . f .=36, P \geq 0.05)$.

\section{Detection of DENV and ZIKV RNA in Ae. aegypti}

Females were sorted into 166 pools and were analyzed for flavivirus RNA by RT-PCR and Sanger sequencing. Two pools were positive. The minimal infection rate (MIR) for female Ae. aegypti was 1.2. One pool contained DENV-1 RNA and the other contained ZIKV RNA. Both pools comprised of mosquitoes collected on day 13 and 17 during the gonotrophic cycle in the rainy season (September 2016). We report some evidence of vertical transmission of DENV-1 in nulliparous females of Ae. aegypti; these females $(\mathrm{n}=11)$ were collected on day 13 in the gonotrophic cycle.

\section{Sequences analyses of DENV-1}

The phylogenetic analysis was performed using 94 DENV-1 sequences (Supplemental Table 1). The sequences correspond to a 204 nucleotides region of the NS5 gene. Many sequences were identical to others and therefore
Table 3 - Correlation indices of the parity rates of Ae. aegypti captured in a cemetery from Merida, Yucatan, during the rainy and dry seasons in 2016, by cross-correlation analysis of a time series

\begin{tabular}{lcc}
\hline Day & $\begin{array}{c}\text { Filtered dates of } \\
\text { the dry season }\end{array}$ & $\begin{array}{c}\text { Crudes dates of } \\
\text { the rainy season }\end{array}$ \\
\hline 0 & 0.69 & 0,02 \\
1 & 0.03 & 0,60 \\
2 & ${ }^{*} 0.02$ & 0,56 \\
3 & 0.00 & 0,01 \\
4 & 0.02 & 0,00 \\
5 & ${ }^{*} 0.38$ & ${ }^{* *} 0,73$ \\
6 & 0.31 & 0,11 \\
7 & 0.14 & 0,43 \\
8 & ${ }^{*} 0.54$ & 0,22 \\
9 & 0.20 & ${ }^{* *} 0,73$ \\
10 & 0.21 & 0,55 \\
11 & - & 0,29 \\
12 & - & 0,14 \\
13 & - & $* 0,64$ \\
14 & - & 0,07 \\
15 & - & 0,69 \\
\hline
\end{tabular}

*High correlation coefficient value appearing every three days in dry season. ${ }^{\star *}$ High correlation coefficient value appearing every four days in rainy season

considered to represent the same "variant". There were eleven variants of DENV-1 (designated DENV-1, V1 to V11). The DENV-1 sequence obtained in this study (V11-DENV-1; Mex 2016) has a close phylogenetic relationship with V1-DENV-1 isolates from Brazil in 2015 with $98.5 \%$ nucleotide identity and similarity. Alignment of 
Table 4 - Parity rate of female Ae. aegypti captured in cemetery from Merida, Yucatan, during the rainy and dry seasons in 2016

\begin{tabular}{|c|c|c|c|c|c|c|c|c|}
\hline \multirow{2}{*}{ Day } & \multicolumn{4}{|c|}{ Dry season } & \multicolumn{4}{|c|}{ Rainy season } \\
\hline & Dissected & Nulliparous & Parous & Parity rate & Dissected & Nulliparous & Parous & Parity rate \\
\hline 1 & 9 & 3 & 6 & 0,67 & 8 & 1 & 7 & 0,88 \\
\hline 2 & 3 & 2 & 1 & 0,33 & 13 & 6 & 7 & 0,54 \\
\hline 3 & 8 & 1 & 7 & 0,88 & 7 & 2 & 5 & 0,71 \\
\hline 4 & 4 & 1 & 3 & 0,75 & 16 & 5 & 11 & 0,69 \\
\hline 5 & 4 & 3 & 1 & 0,25 & 27 & 4 & 23 & 0,85 \\
\hline 6 & 6 & 3 & 3 & 0,50 & 8 & 2 & 6 & 0,75 \\
\hline 7 & 2 & 0 & 2 & 1,00 & 18 & 2 & 16 & 0,89 \\
\hline 8 & 6 & 0 & 6 & 1,00 & 6 & 0 & 6 & 1,00 \\
\hline 9 & 4 & 2 & 2 & 0,50 & 12 & 3 & 9 & 0,75 \\
\hline 10 & 7 & 3 & 4 & 0,57 & 27 & 2 & 25 & 0,93 \\
\hline 11 & 3 & 3 & 0 & 0,00 & 27 & 7 & 20 & 0,74 \\
\hline 12 & 4 & 3 & 1 & 0,25 & 24 & 10 & 14 & 0,58 \\
\hline 13 & 5 & 1 & 4 & 0,80 & 55 & 38 & 17 & 0,31 \\
\hline 14 & 3 & 2 & 1 & 0,33 & 36 & 32 & 4 & 0,11 \\
\hline 15 & 4 & 3 & 1 & 0,25 & 32 & 13 & 19 & 0,59 \\
\hline 16 & 4 & 2 & 2 & 0,50 & 51 & 23 & 28 & 0,55 \\
\hline 17 & 4 & 2 & 2 & 0,50 & 66 & 18 & 48 & 0,73 \\
\hline 18 & 3 & 1 & 2 & 0,67 & 68 & 25 & 43 & 0,63 \\
\hline 19 & 3 & 1 & 2 & 0,67 & 40 & 16 & 24 & 0,60 \\
\hline Total & 86 & 36 & 50 & 0,58 & 541 & 209 & 332 & 0,61 \\
\hline
\end{tabular}

the deduced amino acid sequences revealed that they have $100 \%$ identity and similarity. Likewise, the V11 obtained in this study has a close phylogenetic relationship with V2-DENV-1 identified in Merida, Mexico in 2016 with 99.0\% nucleotide identity and similarity (Supplemental Table 1). Alignment of the deduced amino acid sequences revealed that they have $98.5 \%$ identity and $100 \%$ similarity. The genetic distance Kimura-2 parameter between the $\mathrm{V} 1$ and V2 was 0.015 , while V11 and V2 was 0.01 (Supplemental Table 2). The most common DENV was V8 $(\mathrm{n}=46)$, which was isolated in Mexico, USA, and Nicaragua (Supplemental Table 1).

\section{Sequence analysis of ZIKV}

The phylogenetic analysis was performed using 100 ZIKV sequences (Supplemental Table 1). The sequences correspond to a 172 nucleotides region of the NS5 gene. There were six variants of ZIKV (designated V1 to V6). The ZIKV sequence obtained in this study (V1-ZIKV; Mex 2016) has a close phylogenetic relationship with V2-ZIKV (Asian genotype) isolates from Guatemala (2015), Mexico (2015-2016), China (2016), Honduras (2016), Nicaragua
(2016), Russia (2016-2017) and USA (2016-2017) with 98.3\% nucleotide identity. Alignment of the deduced amino acid sequences revealed that they have $100 \%$ identity and similarity. The genetic distance Kimura-2 parameter between the V1 and V2 was 0.018 (Supplemental Table 3). The most common ZIKV was V2 $(\mathrm{n}=80)$, followed by V3 $(\mathrm{n}=17)$, which was isolated in El Salvador, China, Mexico, Ecuador, Taiwan and Colombia (Supplemental Table 1).

\section{DISCUSSION}

The findings of the present study suggest that the Merida city general cemetery is an important focus of Ae. aegypti proliferation. Vases infestation was high in the present study. An average of 15 infested vases was reported in a quadrant of $100 \mathrm{~m}$, while in Venezuela it was 39 per hectare ${ }^{28}$. Another notable result is that the number of larvae and pupae of Ae. aegypti was high in both seasons. The most likely explanation for the high abundance of mosquitoes and frequency of infested vases during the dry season is in part due to the water supplied by human action as occur in houses ${ }^{29}$. In contrast with this result, in cemeteries from Philippines and Venezuela, 
most vases had water and yielded immature mosquitoes during the rainy season ${ }^{2,28}$. The results of this study suggest that the heterogeneous urban environment supports a high population of mosquitoes. In addition to the general cemetery, previous studies in Merida have also shown that breeding sites on houses, streets/sidewalks and vacant lots yield high number of immature Ae. aegypti ${ }^{29-31}$.

Immature Ae. aegypti was found to be the dominant species in the vases. Ninety-two percent of the vases containing larvae and pupae had only Ae. aegypti. Nevertheless, $C x$. quinquefasciatus, $C x$. coronator and $C x$. nigripalpus were also found. In cemeteries from Philippines and Argentina, Ae. aegypti was found co-inhabiting with Ae. albopictus and $C x$. pipiens, respectively ${ }^{2,3}$. The adaptive features of Ae. aegypti eggs to enter diapause allowed their reproductive success. The diapause may extend for six months or more, until the eggs get in contact with water in the container again, and then hatching occurs ${ }^{32}$. In the cemeteries, it is not possible to control the rain factor, therefore, it is important to have a method to control the presence of larvae and pupae in the vases. In a cemetery of Buenos Aires, Argentina, temephos was effective in reducing Ae. aegypti populations ${ }^{12}$. Meanwhile, Toxorhynchites splendens (Wiedemann) was effective in controlling the larvae of Ae. albopictus in Malaysia $^{13}$.

In the present study, $80 \%(1,327 / 1,648)$ of the Ae. aegypti females were classified as unfed. It is possible that the emerged adults fly towards the nearby houses in search of a blood meal. It is necessary to perform studies on the dispersion of Ae. aegypti from cemeteries to houses, as this will probably limit the efficacy of attempts to suppress the mosquitoes in domestic environments. In contrast to the cemeteries, it is common to find engorged Ae. aegypti in indoor environments. This may be the result of a closer relationship with human ${ }^{15}$. It should be noted that Ae. aegypti display a strong anthropophilia. In houses and schools of Merida city, $57 \%$ of the Ae. aegypti females were collected as fed, $29 \%$ as unfed and $14 \%$ as gravid females $^{11,15}$. In churches, $47 \%$ of the Ae. aegypti females were collected as fed, $34 \%$ as unfed and $19 \%$ as gravid females ${ }^{10}$.

Previous studies on the gonotrophic cycle of Ae. aegypti was estimated with human bait and mark-releaserecapture experiment ${ }^{6,33}$. Currently, human bait is not used due to ethical issues, while the second method requires more effort and sometimes has poorer results. We use BG-Sentinel traps and they turned out to be an effective method for surveillance of Ae. aegypti. In our study, estimated intervals between two consecutive blood meals were three days during dry season and four days during rainy season. The gonotrophic cycle of three days was affected by high temperatures $\left(29.43{ }^{\circ} \mathrm{C}\right)$ during dry season. Under laboratory conditions, high temperatures are significantly more favorable for shorter gonotrophic cycles of Ae. aegypti ${ }^{8}$. Our results agree with previous findings in studies conducted in Thailand ${ }^{33}$, East Africa ${ }^{34}$ and Peru ${ }^{9}$. Additionally, in Thailand, Pant and Yasuno ${ }^{35}$ estimated the gonotrophic cycle of three days during the rainy season, with two days of delay during the dry season. During the rainy season, we estimated a four-day cycle. This result is comparable with the ones from studies performed using the mark-release-recapture method in Thailand ${ }^{35}$, Tanzania ${ }^{36}$ and Kenya ${ }^{37}$. In Merida city, two studies have estimated the gonotrophic cycle of Ae. aegypti. In houses, Rebollar-Tellez et al. ${ }^{6}$ estimated a seven-day cycle, while in churches, the duration of the gonotrophic cycle was similar to the one found in the present study of three and four days during the dry and rainy season, respectively ${ }^{10}$.

High values of survival rate increase the potential risk for transmission of pathogens day to day ${ }^{20}$. Under laboratory conditions, the highest survival rate for Ae. aegypti females was $84 \%$ at $27{ }^{\circ} \mathrm{C}$, reaching 25 days of age ${ }^{8}$. In Mexico, the survivorship for Ae. aegypti was estimated by Rebollar-Tellez et al. ${ }^{6}$ as 0.86 . In the cemetery of Merida city, we found a high survival rate (0.83) for Ae. aegypti. Previous studies conducted in cemeteries identified arbovirus-infected mosquitoes. For example, La Crosse encephalitis virus-infected Aedes triseriatus were collected in cemeteries in Tennessee, USA ${ }^{4}$. In the State of San Luis Potosi, Mexico, ZIKV-infected Ae. aegypti were detected in cemeteries ${ }^{5}$. In the present study, DENV-1 RNA and ZIKV RNA were identified in Ae. aegypti. It is also the first report of Ae. aegypti infected with ZIKV RNA in Yucatan State. Notably, the sequence obtained in this study revealed that the viruses are more closely related phylogenetically to DENV and ZIKV from Central and South America (Supplemental Table 1).The MIR in this study was 1.2 which is considerably lower than the 4.6 reported in schools in Merida ${ }^{11}$. However, our results are similar to the ones from earlier studies performed inside the houses of dengue patients ${ }^{15,38}$. On the other hand, the first report of ZIKVinfected Ae. aegypti was from Chiapas, Mexico and the MIR was estimated at 52.49-172.66

We also found evidence of vertical transmission of DENV-1 in nulliparous Ae. aegypti females during the gonotrophic cycle. In Mexico, vertical transmission of dengue virus by Ae. aegypti and Ae. albopictus was reviewed by Ferreira-de-Lima and Lima-Camara ${ }^{40}$, who mentioned that they occur in Tamaulipas, Oaxaca and Guerrero. Vertical transmission may represent an important strategy for maintaining the circulation of arboviruses in nature ${ }^{40}$, therefore it should be studied in depth in the cemeteries. 


\section{CONFLICT OF INTERESTS}

None.

\section{FUNDING}

The study was supported by the Consejo Nacional de Ciencia y Tecnologia de Mexico, Grant Problemas Nacionales PDCPN 2014-247005.

\section{ACKNOWLEDGMENTS}

We thank the laboratory staff at Arbovirologia of Universidad Autonoma de Yucatan for assistance with mosquitoes collection.

\section{AUTHORS' CONTRIBUTIONS}

J. Garcia-Rejon and C. M Baak-Baak conceived and designed the study; N. Cigarroa-Toledo and A. Pech-May carried out the phylogenetic analysis; R. C. Cetina-Trejo, L. G. Talavera-Aguilar, and O. M. Torres-Chable carried out the fieldwork and the labwork; A. Ulloa-Garcia, C. MachainWilliams, and J. C. Navarro analyzed the data. All authors contributed for drafted the manuscript, provided critical input regarding the findings and approved the final manuscript.

\section{REFERENCES}

1. Vezzani D. Review: artificial container-breeding mosquitoes and cemeteries: a perfect match. Trop Med Int Health. 2007;12:299-313.

2. Schultz GW. Cemetery vase breeding of dengue vectors in Manila, Republic of the Philippines. J Am Mosq Control Assoc. 1989;5:508-13

3. Vezzani D, Albicócco AP. The effect of shade on the container index and pupal productivity of the mosquitoes Aedes aegypti and Culex pipiens breeding in artificial containers. Med Vet Entomol. 2009;23:8-84.

4. Trout Fryxell RT, Freyman K, Ulloa A, Hendricks B, Paulsen D, Odoi A, et al. Cemeteries are effective sites for monitoring la crosse virus ( $\mathrm{LACv}$ ) and these environments may play a role in LACv infection. PLoS One. 2015;10:e0122895.

5. Huerta H, González-Roldán JF, Sánchez-Tejeda G, CorreaMorales F, Romero-Contreras FE, Cárdenas-Flores R, et al Detection of Zika virus in Aedes mosquitoes from Mexico. Trans R Soc Trop Med Hyg. 2017;111:328-31.

6. Rebollar-Téllez EA, Loroño-Pino MA, Rodríguez-Angulo EM, Farfán-Ale JA. Blood-feeding frequency and life expectancy of Aëdes aegypti (Diptera: Culicidae) in an urban area of Merida city, state of Yucatan, Mexico. Rev Biomed. 1995;6:135-41.
7. Birley MH, Rajagopalan PK. Estimation of the survival and biting rates of Culex quinquefasciatus (Diptera: Culicidae). J Med Entomol. 1981;18:181-6.

8. Goindin D, Delannay C, Ramdini C, Gustave J, Fouque F. Parity and longevity of Aedes aegypti according to temperatures in controlled conditions and consequences on dengue transmission risks. PLoS One. 2015;10:e135489.

9. Wong J, Astete H, Morrison AC, Scott TW. Sampling considerations for designing Aedes aegypti (Diptera:Culicidae) oviposition studies in Iquitos, Peru: substrate preference, diurnal periodicity, and gonotrophic cycle length. J Med Entomolol. 2011;48:45-52

10. Baak-Baak CM, Ulloa-Garcia A, Cigarroa-Toledo N, Tzuc Dzul JC, Machain-Williams C, Torres-Chable OM, et al. Blood feeding status, gonotrophic cycle and survivorship of Aedes (Stegomyia) aegypti (L.) (Diptera: Culicidae) caught in churches from Merida, Yucatan, Mexico. Neotrop Entomol. 2017;46:622-30.

11. García-Rejón JE, Loroño-Pino MA, Farfán-Ale JA, Flores-Flores LF, López-Uribe MP, Najera-Vazquez MR, et al. Mosquito infestation and dengue virus infection in Aedes aegypti females in schools in Merida, Mexico. Am J Trop Med Hyg. 2011;84:489-96.

12. Vezzani D, Velázquez SM, Schweigmann N. Control of Aedes aegypti with temephos in a Buenos Aires cemetery, Argentina. Rev Saude Publica. 2004;38:738-40.

13. Nyamah MA, Sulaiman S, Omar B. Field observation on the efficacy of Toxorhynchites splendens (Wiedemann) as a biocontrol agent against Aedes albopictus (Skuse) larvae in a cemetery. Trop Biomed. 2011;28:312-9.

14. Salomón-Grajales J, Lugo-Moguel GV, Tinal-Gordillo VR, de La Cruz-Velázquez J, Beaty BJ, Eisen L, et al. Aedes albopictus Mosquitoes, Yucatan Peninsula, Mexico. Emerg Infect Dis. 2012;18:525-7.

15. Garcia-Rejon J, Loroño-Pino MA, Farfan-Ale JA, Flores-Flores L, Del Pilar Rosado-Paredes E, Rivero-Cardenas N, et al. Dengue virus-infected Aedes aegypti in the home environment. Am J Trop Med Hyg. 2008;79:940-50.

16. Cigarroa-Toledo N, Blitvich BJ, Cetina-Trejo RC, TalaveraAguilar LG, Baak-Baak CM, Torres-Chablé OM, et al. Chikungunya virus in febrile humans and Aedes aegypti mosquitoes, Yucatan, Mexico. Emerg Infect Dis. 2016;22:18047

17. Carpenter SJ, LaCasse WJ. Mosquitoes of North America (North of Mexico). Berkeley: University of California Press; 1955.

18. Darsie RF, Ward RA. Identification and geographical distribution of the mosquitoes of North America, North of Mexico. Gainesville: University Press of Florida; 2005.

19. Detinova TS. Age-grouping methods in Diptera of medical importance: with special reference to some vectors of malaria. Geneva: World Health Organization; 1962. 
20. Scaramozzino N, Crance JM, Jouan A, DeBriel DA, Stoll F, Garin D. Comparison of flavivirus universal primer pairs and development of a rapid, highly sensitive heminested reverse transcription-PCR assay for detection of flaviviruses targeted to a conserved region of the NS5 gene sequences. J Clin Microbiol. 2001; 39:1922-7.

21. Holmes PR, Birley MH. An improved method for survival rate analysis from time series of haematophagous dipteran populations. J Animal Ecol. 1987;56:427-40.

22. Davidson G. Estimation of the survival-rate of anopheline mosquitoes in nature. Nature. 1954;174:792-3.

23. Hall T. BioEdit: an important software for molecular biology. GERF Bull Biosci. 2011;2:60-1.

24. Kumar S, Stecher G, Tamura K. MEGA7: Molecular Evolutionary Genetics Analysis version 7.0 for bigger datasets. Mol Biol Evol. 2016;33:1870-4.

25. Librado P, Rozas J. DnaSP v5: a software for comprehensive analysis of DNA polymorphism data. Bioinformatics. 2009;25:1451-2

26. Campanella JJ, Bitincka L, Smalley J. MatGAT: an application that generates similarity/identity matrices using protein or DNA sequences. BMC Bioinformatics. 2003;4:29.

27. Bockarie MJ, Service MW, Barnish G, Touré YT. Vectorial capacity and entomological inoculation rates of Anopheles gambiae in a high rainfall forested area of southern Sierra Leone. Trop Med Parasitol. 1995;46:164-71.

28. Abe M, McCall PJ, Lenhart A, Villegas E, Kroeger A. The Buen Pastor cemetery in Trujillo, Venezuela: measuring dengue vector output from a public area. Trop Med Int Health. 2005; 10:597-603.

29. García-Rejón JE, López-Uribe MP, Loroño-Pino MA, FarfánAle JA, Del Najera-Vazquez MR, Lozano-Fuentes S, et al Productive container types for Aedes aegypti immatures in Merida, Mexico. J Med Entomol. 2011;48:644-50.

30. Baak-Baak CM, Arana-Guardia R, Cigarroa-Toledo N, LoroñoPino MA, Reyes-Solis G, Machain-Williams C, et al. Vacant lots: productive sites for Aedes (Stegomyia) aegypti (Diptera: Culicidae) in Merida City, Mexico. J Med Entomol. 2014;51:475-83
31. Arana-Guardia R, Baak-Baak CM, Loroño-Pino MA, MachainWilliams C, Beaty BJ, Eisen L, et al. Stormwater drains and catch basins as sources for production of Aedes aegypti and Culex quinquefasciatus. Acta Trop. 2014;134:33-42.

32. Soares-Pinheiro VC, Dasso-Pinheiro W, Trindade-Bezerra JM, Tadei WP. Eggs viability of Aedes aegypti Linnaeus (Diptera, Culicidae) under different environmental and storage conditions in Manaus, Amazonas, Brazil. Braz J Biol. 2017;77:396-401.

33. Sheppard PM, Macdonald WW, Tonn RJ, Grab B. The dynamics of an adult population of Aedes aegypti in relation to dengue haemorrhagic fever in Bangkok. J Animal Ecol. 1969;38:661702 .

34. McClelland GA, Conway GR. Frequency of blood feeding in the mosquito Aedes aegypti. Nature. 1971;232:485-6.

35. Pant CP, Yasuno M. Field studies on the gonotrophic cycle of Aedes aegypti in Bangkok, Thailand. J Med Entomol. 1973;10:219-23.

36. Conway GR, Trpis M, McClelland GA. Population parameters of the mosquito Aedes aegypti (L.) estimated by mark-releaserecapture in a suburban habitat in Tanzania. J Animal Ecol. 1974;43:289-304

37. Trpis M, Hausermann W. Dispersal and other population parameters of Aedes aegypti in an African village and their possible significance in epidemiology of vector-borne diseases. Am J Trop Med Hyg. 1986;35:1263-79.

38. Eisen L, Garcia-Rejón JE, Gómez-Carro S, Nájera Vázquez MR, Keefe TJ, Beaty BJ, et al. Temporal correlations between mosquito-based dengue virus surveillance measures or indoor mosquito abundance and dengue case numbers in Merida City, Mexico. Mexico. J Med Entomol. 2014;51:885-90.

39. Guerbois M, Fernandez-Salas I, Azar SR, Danis-Lozano R, Alpuche-Aranda CM, Leal G, et al. Outbreak of Zika virus infection, Chiapas State, Mexico, 2015, and first confirmed transmission by Aedes aegypti mosquitoes in the Americas. $\mathrm{J}$ Infect Dis. 2016; 214:1349-56.

40. Ferreira-de-Lima VH, Lima-Camara TN. Natural vertical transmission of dengue virus in Aedes aegypti and Aedes albopictus: a systematic review. Parasit Vectors. 2018;11:77. 
Supplemental Table 1 - Database with GenBank accession numbers

\begin{tabular}{|c|c|c|c|c|c|c|}
\hline $\begin{array}{l}\text { GenBank accession } \\
\text { number }\end{array}$ & $\begin{array}{l}\text { Clave in } \\
\text { the study }\end{array}$ & Virus & Genotype & Collection date & State & Country \\
\hline KU232287 & $\mathrm{V} 1$ & Dengue & 1 & 2015 & Pernambuco & Brazil \\
\hline KU232286 & V1 & Dengue & 1 & 2015 & Pernambuco & Brazil \\
\hline Merida, Mex-2016 & V2 & Dengue & & 2016 & Yucatan & Mexico \\
\hline KF973475 & V3 & Dengue & & 2012 & No data & Nicaragua \\
\hline KF973472 & V3 & Dengue & & 2012 & No data & Nicaragua \\
\hline KF973467 & V3 & Dengue & & 2012 & No data & Nicaragua \\
\hline KF973466 & V3 & Dengue & & 2012 & No data & Nicaragua \\
\hline KF973463 & V3 & Dengue & & 2012 & No data & Nicaragua \\
\hline KF973460 & V3 & Dengue & & 2012 & No data & Nicaragua \\
\hline KF973458 & V3 & Dengue & & 2012 & No data & Nicaragua \\
\hline KF973456 & V3 & Dengue & & 2012 & No data & Nicaragua \\
\hline KF973455 & V3 & Dengue & & 2012 & No data & Nicaragua \\
\hline KF973454 & V3 & Dengue & & 2012 & No data & Nicaragua \\
\hline KJ189349 & V3 & Dengue & & 2011 & Yucatan & Mexico \\
\hline KJ189348 & V3 & Dengue & & 2011 & Yucatan & Mexico \\
\hline GQ199859 & V3 & Dengue & & 2008 & Managua & Nicaragua \\
\hline KJ189342 & V4 & Dengue & & 2009 & Yucatan & Mexico \\
\hline KJ189341 & V4 & Dengue & & 2009 & Yucatan & Mexico \\
\hline KF973474 & V5 & Dengue & & 2012 & No data & Nicaragua \\
\hline KF973473 & V6 & Dengue & & 2012 & No data & Nicaragua \\
\hline KJ189359 & V7 & Dengue & & 2012 & No data & Puerto Rico \\
\hline KJ189345 & V8 & Dengue & & 2009 & Yucatan & Mexico \\
\hline KJ189343 & V8 & Dengue & & 2009 & Yucatan & Mexico \\
\hline KJ189339 & V8 & Dengue & & 2008 & Yucatan & Mexico \\
\hline KJ189337 & V8 & Dengue & & 2008 & Yucatan & Mexico \\
\hline KJ189333 & V8 & Dengue & & 2008 & Yucatan & Mexico \\
\hline KJ189332 & V8 & Dengue & & 2008 & Yucatan & Mexico \\
\hline KJ189331 & V8 & Dengue & & 2008 & Yucatan & Mexico \\
\hline KJ189321 & V8 & Dengue & & 2007 & Yucatan & Mexico \\
\hline KJ189320 & V8 & Dengue & & 2007 & Yucatan & Mexico \\
\hline KJ189319 & V8 & Dengue & & 2007 & Yucatan & Mexico \\
\hline KJ189318 & V8 & Dengue & & 2007 & Yucatan & Mexico \\
\hline KJ189313 & V8 & Dengue & & 2008 & Yucatan & Mexico \\
\hline KF955443 & V8 & Dengue & & 2007 & Yucatan & Mexico \\
\hline KF955442 & V8 & Dengue & & 2007 & Yucatan & Mexico \\
\hline KF955433 & V8 & Dengue & & 2008 & Yucatan & Mexico \\
\hline KF955422 & V8 & Dengue & & 2007 & Yucatan & Mexico \\
\hline JQ675358 & V8 & Dengue & & 2010 & Florida & USA \\
\hline JQ287666 & V8 & Dengue & & 2009 & Managua & Nicaragua \\
\hline JN819403 & V8 & Dengue & & 2006 & Managua & Nicaragua \\
\hline JN819402 & V8 & Dengue & & 2005 & Managua & Nicaragua \\
\hline JF937644 & V8 & Dengue & & 2009 & Managua & Nicaragua \\
\hline JF937645 & V8 & Dengue & & 2009 & Managua & Nicaragua \\
\hline HM631855 & V8 & Dengue & & 2007 & Yucatan & Mexico \\
\hline GU131976 & V8 & Dengue & & 2007 & Yucatan & Mexico \\
\hline GU131968 & V8 & Dengue & & 2007 & Yucatan & Mexico \\
\hline GU131966 & V8 & Dengue & & 2007 & Yucatan & Mexico \\
\hline GU131964 & V8 & Dengue & & 2007 & Yucatan & Mexico \\
\hline GU131961 & V8 & Dengue & & 2007 & Yucatan & Mexico \\
\hline GU131960 & V8 & Dengue & & 2007 & Yucatan & Mexico \\
\hline
\end{tabular}


Supplemental Table 1 - Database with GenBank accession numbers (cont.)

\begin{tabular}{|c|c|c|c|c|c|c|}
\hline $\begin{array}{l}\text { GenBank accession } \\
\text { number }\end{array}$ & $\begin{array}{l}\text { Clave in } \\
\text { the study }\end{array}$ & Virus & Genotype & Collection date & State & Country \\
\hline GU131958 & V8 & Dengue & & 2006 & Yucatan & Mexico \\
\hline GQ868539 & V8 & Dengue & & 2008 & Yucatan & Mexico \\
\hline GQ868536 & V8 & Dengue & & 2008 & Yucatan & Mexico \\
\hline GQ868527 & V8 & Dengue & & 2007 & Yucatan & Mexico \\
\hline GQ868509 & V8 & Dengue & & 2007 & Yucatan & Mexico \\
\hline GQ868503 & V8 & Dengue & & 2007 & Yucatan & Mexico \\
\hline GQ868501 & V8 & Dengue & & 2007 & Yucatan & Mexico \\
\hline GQ868499 & V8 & Dengue & & 2006 & Quintana Roo & Mexico \\
\hline GQ868498 & V8 & Dengue & & 2006 & Yucatan & Mexico \\
\hline GQ199875. & V8 & Dengue & & 2004 & Managua & Nicaragua \\
\hline GQ199873 & V8 & Dengue & & 2004 & Managua & Nicaragua \\
\hline GQ199872 & V8 & Dengue & & 2004 & Managua & Nicaragua \\
\hline GQ199867 & V8 & Dengue & & 2004 & Managua & Nicaragua \\
\hline FJ898433 & V8 & Dengue & & 2007 & Managua & Nicaragua \\
\hline FJ873814 & V8 & Dengue & & 2005 & Managua & Nicaragua \\
\hline FJ850114 & V8 & Dengue & & 2005 & Managua & Nicaragua \\
\hline FJ850113 & V8 & Dengue & & 2005 & Managua & Nicaragua \\
\hline KF955408 & V9 & Dengue & & 2007 & No data & Venezuela \\
\hline KF955407 & V9 & Dengue & & 2005 & No data & Venezuela \\
\hline JN819415 & V9 & Dengue & & 2006 & Aragua & Venezuela \\
\hline JN819413 & V9 & Dengue & & 2006 & Aragua & Venezuela \\
\hline JN819412 & V9 & Dengue & & 2006 & Aragua & Venezuela \\
\hline JN819411 & V9 & Dengue & & 2005 & Aragua & Venezuela \\
\hline JN819405 & V9 & Dengue & & 2006 & Merida & Venezuela \\
\hline GU131842 & V9 & Dengue & & 2007 & Aragua & Venezuela \\
\hline GQ868570 & V9 & Dengue & & 2008 & Santander & Colombia \\
\hline GQ868562 & V9 & Dengue & & 2005 & Santander & Colombia \\
\hline FJ882579 & V9 & Dengue & & 2007 & Aragua & Venezuela \\
\hline FJ873809 & V9 & Dengue & & 2007 & Aragua & Venezuela \\
\hline FJ850101 & V9 & Dengue & & 2007 & Aragua & Venezuela \\
\hline FJ850100 & V9 & Dengue & & 2007 & Aragua & Venezuela \\
\hline FJ850099 & V9 & Dengue & & 2007 & Aragua & Venezuela \\
\hline FJ850093 & V9 & Dengue & & 2008 & No data & Brazil \\
\hline FJ639824 & V9 & Dengue & & 2006 & Aragua & Venezuela \\
\hline FJ639823 & V9 & Dengue & & 2006 & Aragua & Venezuela \\
\hline FJ639820 & V9 & Dengue & & 2006 & Aragua & Venezuela \\
\hline FJ639818 & V9 & Dengue & & 2006 & Aragua & Venezuela \\
\hline FJ639813 & V9 & Dengue & & 2005 & Aragua & Venezuela \\
\hline FJ639812 & V9 & Dengue & & 2005 & Aragua & Venezuela \\
\hline FJ639802 & V9 & Dengue & & 2005 & Aragua & Venezuela \\
\hline FJ639796 & V9 & Dengue & & 2005 & Aragua & Venezuela \\
\hline GU056032 & V10 & Dengue & & 1998 & Aragua & Venezuela \\
\hline FJ898437 & V10 & Dengue & & 2004 & Managua & Nicaragua \\
\hline At present study & V11 & Dengue & & 2016 & Yucatan & Mexico \\
\hline At present study & V1 & Zika & Asian & 2016 & Yucatan & Mexico \\
\hline MF801426 & V2 & Zika & & 2016 & No data & Nicaragua \\
\hline MF801424 & V2 & Zika & & 2016 & Yucatan & Mexico \\
\hline MF801423 & V2 & Zika & & 2016 & Guerrero & Mexico \\
\hline MF801422 & V2 & Zika & & 2016 & Guerrero & Mexico \\
\hline MF801420 & V2 & Zika & & 2016 & Chiapas & Mexico \\
\hline
\end{tabular}


Supplemental Table 1 - Database with GenBank accession numbers (cont.)

\begin{tabular}{|c|c|c|c|c|c|c|}
\hline $\begin{array}{l}\text { GenBank accession } \\
\text { number }\end{array}$ & $\begin{array}{l}\text { Clave in } \\
\text { the study }\end{array}$ & Virus & Genotype & Collection date & State & Country \\
\hline MF801418 & V2 & Zika & & 2016 & Chiapas & Mexico \\
\hline MF801417 & V2 & Zika & & 2016 & Chiapas & Mexico \\
\hline MF801414 & V2 & Zika & & 2016 & Guerrero & Mexico \\
\hline MF801413 & V2 & Zika & & 2016 & Guerrero & Mexico \\
\hline MF801412 & V2 & Zika & & 2016 & Guerrero & Mexico \\
\hline MF801411 & V2 & Zika & & 2016 & Guerrero & Mexico \\
\hline MF801410 & V2 & Zika & & 2016 & Guerrero & Mexico \\
\hline MF801408 & V2 & Zika & & 2016 & Chiapas & Mexico \\
\hline MF801406 & V2 & Zika & & 2016 & Oaxaca & Mexico \\
\hline MF801405 & V2 & Zika & & 2016 & Guerrero & Mexico \\
\hline MF801403 & V2 & Zika & & 2016 & Chiapas & Mexico \\
\hline MF801402 & V2 & Zika & & 2016 & Chiapas & Mexico \\
\hline MF801401 & V2 & Zika & & 2016 & Chiapas & Mexico \\
\hline MF801400 & V2 & Zika & & 2016 & Chiapas & Mexico \\
\hline MF801399 & V2 & Zika & & 2016 & Chiapas & Mexico \\
\hline MF801398 & V2 & Zika & & 2016 & Chiapas & Mexico \\
\hline MF801396 & V2 & Zika & & 2016 & Chiapas & Mexico \\
\hline MF801395 & V2 & Zika & & 2016 & Chiapas & Mexico \\
\hline MF801391 & V2 & Zika & & 2016 & Oaxaca & Mexico \\
\hline MF801389 & V2 & Zika & & 2016 & Roatan & Honduras \\
\hline MF801387 & V2 & Zika & & 2016 & Roatan & Honduras \\
\hline MF801386 & V2 & Zika & & 2016 & Roatan & Honduras \\
\hline MF801385 & V2 & Zika & & 2016 & Roatan & Honduras \\
\hline MF801384 & V2 & Zika & & 2016 & Roatan & Honduras \\
\hline MF801383 & V2 & Zika & & 2016 & No data & Honduras \\
\hline MF801377 & V3 & Zika & & 2016 & No data & EI Salvador \\
\hline KX906952 & V2 & Zika & & 2016 & No data & Honduras \\
\hline MF593625 & V2 & Zika & Asian & 2016 & No data & China \\
\hline MF434522 & V2 & Zika & Asian & 2016 & Managua & Nicaragua \\
\hline MF434521 & V2 & Zika & Asian & 2016 & Managua & Nicaragua \\
\hline MF434517 & V2 & Zika & Asian & 2016 & Managua & Nicaragua \\
\hline MF434516 & V2 & Zika & Asian & 2016 & Managua & Nicaragua \\
\hline MF159531 & V2 & Zika & & 2017 & Miami & USA \\
\hline MF098771 & V2 & Zika & & 2017 & No data & Russia \\
\hline MF098770 & V2 & Zika & & 2016 & No data & Russia \\
\hline KY927808 & V2 & Zika & & 2016 & Henan & China \\
\hline KY765327 & V2 & Zika & & 2016 & Managua & Nicaragua \\
\hline KY765326 & V2 & Zika & & 2016 & Managua & Nicaragua \\
\hline KY765325 & V2 & Zika & & 2016 & Managua & Nicaragua \\
\hline KY765324 & V2 & Zika & & 2016 & Managua & Nicaragua \\
\hline KY765323 & V2 & Zika & & 2016 & Managua & Nicaragua \\
\hline KY765320 & V2 & Zika & & 2016 & Managua & Nicaragua \\
\hline KY785461 & V2 & Zika & & 2016 & Francisco Morazan & Honduras \\
\hline KY785457 & V2 & Zika & & 2016 & Florida & USA \\
\hline KY785452 & V2 & Zika & & 2016 & Francisco Morazan & Honduras \\
\hline KY785442 & V2 & Zika & & 2016 & Francisco Morazan & Honduras \\
\hline KY785431 & V2 & Zika & & 2016 & Francisco Morazan & Honduras \\
\hline KY785418 & V2 & Zika & & 2016 & Francisco Morazan & Honduras \\
\hline KY785414 & V2 & Zika & & 2016 & Francisco Morazan & Honduras \\
\hline KY693677 & V2 & Zika & & 2016 & No data & Honduras \\
\hline
\end{tabular}


Supplemental Table 1 - Database with GenBank accession numbers (cont.)

\begin{tabular}{|c|c|c|c|c|c|c|}
\hline $\begin{array}{l}\text { GenBank accession } \\
\text { number }\end{array}$ & $\begin{array}{l}\text { Clave in } \\
\text { the study }\end{array}$ & Virus & Genotype & Collection date & State & Country \\
\hline KY693676 & V2 & Zika & & 2016 & No data & Honduras \\
\hline KY631494 & V2 & Zika & & 2015 & Tapachula, Chiapas & Mexico \\
\hline KY631493 & V2 & Zika & & 2015 & Tapachula, Chiapas & Mexico \\
\hline KY648934 & V2 & Zika & & 2016 & Chiapas & Mexico \\
\hline KY014327 & V2 & Zika & & 2016 & Francisco Morazan & Honduras \\
\hline KY014319 & V2 & Zika & & 2016 & Francisco Morazan & Honduras \\
\hline KY014315 & V2 & Zika & & 2016 & Francisco Morazan & Honduras \\
\hline KY014312 & V2 & Zika & & 2016 & Francisco Morazan & Honduras \\
\hline KY014310 & V2 & Zika & & 2016 & Francisco Morazan & Honduras \\
\hline KY014306 & V2 & Zika & & 2016 & Francisco Morazan & Honduras \\
\hline KY606274 & V2 & Zika & & 2016 & Guerrero & Mexico \\
\hline KY606273 & V2 & Zika & & 2016 & Guerrero & Mexico \\
\hline KY606272 & V2 & Zika & & 2016 & Oaxaca & Mexico \\
\hline KY606271 & V2 & Zika & & 2016 & Chiapas & Mexico \\
\hline KX421195 & V2 & Zika & & 2016 & No data & Nicaragua \\
\hline KX421194 & V2 & Zika & & 2016 & No data & Nicaragua \\
\hline KY325479 & V2 & Zika & & 2016 & Florida & USA \\
\hline KY325465 & V2 & Zika & & 2016 & Florida & USA \\
\hline KY328289 & V2 & Zika & & 2016 & No data & Honduras \\
\hline KX694534 & V2 & Zika & & 2016 & No data & Honduras \\
\hline KX856011 & V2 & Zika & Asian & 2016 & Chiapas & Mexico \\
\hline KX262887 & V2 & Zika & & 2016 & No data & Honduras \\
\hline KU870645 & V2 & Zika & & 2016 & No data & USA \\
\hline KU501217 & V2 & Zika & & 2015 & No data & Guatemala \\
\hline KU501216 & V2 & Zika & & 2015 & No data & Guatemala \\
\hline MF099651 & V3 & Zika & & 2016 & Guizhou & China \\
\hline MF801421 & V3 & Zika & & 2016 & Chiapas & Mexico \\
\hline MF801419 & V4 & Zika & & 2016 & Chiapas & Mexico \\
\hline MF801397 & V5 & Zika & & 2016 & Campeche & Mexico \\
\hline MF801381 & V6 & Zika & & 2016 & No data & Honduras \\
\hline MF794971 & V3 & Zika & & 2016 & No data & Ecuador \\
\hline MF692778 & V3 & Zika & & 2016 & No data & Taiwan \\
\hline MF574588 & V3 & Zika & & 2016 & Barranquilla & Colombia \\
\hline MF574587 & V3 & Zika & & 2016 & Barranquilla & Colombia \\
\hline MF574586 & V3 & Zika & & 2016 & Barranquilla & Colombia \\
\hline MF574585 & V3 & Zika & Asian & 2016 & Barranquilla & Colombia \\
\hline MF574584 & V3 & Zika & & 2016 & Barranquilla & Colombia \\
\hline MF574583 & V3 & Zika & Asian & 2016 & Barranquilla & Colombia \\
\hline MF574582 & V3 & Zika & & 2016 & Barranquilla & Colombia \\
\hline MF574581 & V3 & Zika & & 2016 & Barranquilla & Colombia \\
\hline MF574580 & V3 & Zika & & 2016 & Barranquilla & Colombia \\
\hline MF574577 & V3 & Zika & & 2016 & Barranquilla & Colombia \\
\hline MF574576 & V3 & Zika & & 2016 & Barranquilla & Colombia \\
\hline MF574575 & V3 & Zika & Asian & 2015 & Barranquilla & Colombia \\
\hline MF574574 & V3 & Zika & & 2015 & Barranquilla & Colombia \\
\hline
\end{tabular}


Supplemental Table 2 - Genetic distance (Kimura 2 parameter model) among the different variants of DENV1 (below the diagonal) and the standard error among variants (above the diagonal)

\begin{tabular}{lccccccccccc}
\hline & V1 & V2 & V3 & V4 & V5 & V6 & V7 & V8 & V9 & V10 & V11 \\
\hline V1 & & 0.008 & 0.012 & 0.01 & 0.013 & 0.013 & 0.008 & 0.011 & 0.011 & 0.011 & 0.011 \\
V2 & 0.015 & & 0.011 & 0.011 & 0.012 & 0.012 & 0.012 & 0.012 & 0.012 & 0.012 & 0.007 \\
V3 & 0.03 & 0.025 & & 0.007 & 0.005 & 0.005 & 0.008 & 0.007 & 0.005 & 0.005 & 0.013 \\
V4 & 0.02 & 0.025 & 0.01 & & 0.008 & 0.008 & 0.008 & 0.005 & 0.008 & 0.005 & 0.013 \\
V5 & 0.035 & 0.03 & 0.005 & 0.015 & & 0.007 & 0.01 & 0.008 & 0.007 & 0.007 & 0.014 \\
V6 & 0.035 & 0.03 & 0.005 & 0.015 & 0.01 & & 0.01 & 0.008 & 0.007 & 0.007 & 0.014 \\
V7 & 0.015 & 0.03 & 0.015 & 0.015 & 0.02 & 0.02 & & 0.008 & 0.007 & 0.007 & 0.014 \\
V8 & 0.025 & 0.03 & 0.01 & 0.005 & 0.015 & 0.015 & 0.015 & & 0.008 & 0.005 & 0.014 \\
V9 & 0.025 & 0.03 & 0.005 & 0.015 & 0.01 & 0.01 & 0.01 & 0.015 & & 0.007 & 0.014 \\
V10 & 0.025 & 0.03 & 0.005 & 0.005 & 0.01 & 0.01 & 0.01 & 0.005 & 0.01 & & 0.014 \\
V11 & 0.025 & 0.01 & 0.035 & 0.035 & 0.04 & 0.04 & 0.04 & 0.04 & 0.04 & 0.04 & \\
\hline V1:
\end{tabular}

V1: DENV-1, Brazil (2015); V2: DENV-1, Merida, Mexico (2016); V11-DENV-1; Mexico (2016 - at present study)

Supplemental Table 3 - Genetic distance (Kimura 2 parameter model) among the different variants of zika virus (below the diagonal) and the standard error among variants (above the diagonal)

\begin{tabular}{lllllll}
\hline & V1 & V2 & V3 & V4 & V5 & V6 \\
\hline V1 & & 0.01 & 0.012 & 0.012 & 0.012 & 0.011 \\
V2 & 0.018 & & 0.006 & 0.006 & 0.006 & 0.006 \\
V3 & 0.024 & 0.006 & & 0.008 & 0.008 & 0.008 \\
V4 & 0.024 & 0.006 & 0.012 & & 0.008 & 0.008 \\
V5 & 0.024 & 0.006 & 0.012 & 0.012 & & 0.008 \\
V6 & 0.024 & 0.006 & 0.012 & 0.012 & 0.012 & \\
\hline
\end{tabular}

V1: V1ZIKV; Mexico (2016 - at present study); V2: ZIKV; Guatemala (2015), Mexico (2015-2016), China (2016), Honduras (2016), Nicaragua (2016), Russia (2016-2017) and USA (2016-2017) 